\title{
The influence of steroid metabolism on CYP17A1 inhibitor activity
}

\section{Frank Claessens and Lisa Moris}

\begin{abstract}
Galeterone is a steroid 17- $\alpha$-hydroxylase/17,20 lyase and androgen receptor antagonist intended for patients with prostate cancer. Similar to abiraterone, galeterone has a steroid scaffold structure and mimics natural ligands; thus, these agents are metabolized by the same enzymes that synthesize or degrade naturally occurring steroids, which can result in attenuated efficacy.
\end{abstract}

Refers to Alyamani, M. et al. Steroidogenic metabolism of galeterone reveals a diversity of biochemical activities. Cell Chem. Biol. 24, 825-832.e6 (2017)

A new study by Alyamani et al. ${ }^{1}$ shows that the steroidal structure of the CYP17A1 inhibitor galeterone makes it susceptible to conversion into metabolites with differing activities on the androgen signalling pathway. The metabolic pathway of abiraterone, another CYP17A1 inhibitor, was previously reported by the same group ${ }^{2}$. Although these two agents have a similar chemical structure, their activity and that of their metabolites differ. The presence of active metabolites with possibly undesirable effects might be a universal drawback of these steroidal drugs.

The androgen receptor (AR) is a survival factor for normal, as well as malignant, prostate cells, and several options to therapeutically target the AR signalling pathway have been exploited in patients with prostate cancer. As a first option in the hormonal treatment of prostate cancer, androgen deprivation is obtained by interfering with the hypothalamic-pituitary-gonadal axis. Luteinizing-hormone-releasing hormone agonists, such as leuprolide or goserelin, are used to inhibit the testicular and adrenal production of androgens, resulting in slowed or halted tumour growth. The cancer eventually becomes independent of these extratumoral androgens and the prostate cancer cells start to grow again, leading to castration-resistant prostate cancer (CRPC). This castration resistance can be caused by AR overexpression and a concomitant rise in expression of AR splice variants (through mutations in $A R$ leading to mutant or truncated receptor versions), by alterations in AR co-regulators or pioneering factors that assist in changing the chromatin status of androgen target genes, by activation of alternative signalling pathways that affect androgen signalling, or by ectopic, intratumoral synthesis of androgens.

The AR signalling axis is still therapeutically targeted in patients with CRPC, either with flutamide-like AR antagonists, such as enzalutamide or apalutamide, or with inhibitors of the androgen biosynthesis. Owing to the importance of the remaining or intratumoral androgen synthesis in CRPC, inhibition of key enzymes in the androgen biosynthesis pathway has been developed as a therapeutic approach ${ }^{3}$. As such inhibitors mimic natural ligands, steroid scaffolds are excellent starting points for their development. However, this resemblance can also be a major drawback, as it might make them susceptible to modifications by the same metabolic enzymes that are responsible for the synthesis or the clearance of naturally occurring steroids ${ }^{4}$.

Abiraterone is the most commonly used inhibitor of steroid 17-a-hydroxylase/17,20 lyase (CYP17A1), which is the key enzyme in androgen biosynthesis, converting pregnenolone into the testosterone precursor dehydroepiandrosterone. Abiraterone is currently in clinical use for the treatment of metastatic CRPC ${ }^{5}$. Galeterone is another steroidal CYP17A1 antagonist and is 2.7-fold more potent in inhibiting the C17,20-lyase activity of CYP17A1 in vitro than abiraterone ${ }^{6}$. Galeterone also acts as an AR antagonist, inducing degradation of wild-type and mutant $\mathrm{ARs}^{7}$. Unfortunately, the phase III clinical trial in which galeterone was compared with enzalutamide in patients with metastatic CRPC positive for expression of the AR splice variant AR-V7 (ARMOR3-SV) was discontinued last year because of the unlikelihood of meeting its trial end point.

This paper by Nima Sharifi's group ${ }^{1}$ gives important new insights into the metabolism of galeterone in preclinical models. In human LNCaP prostate cancer cells, $3 \beta$-hydroxysteroid dehydrogenase ( $3 \beta$-HSD) converts galeterone to $\Delta 4$-galeterone (D4G), which is further converted by steroid $5 a$-reductase (SRD5A) to 3-keto-5a-galeterone $(5 \alpha G)$, which can be further converted to the inactive $3 a-\mathrm{OH}-5 a$-galeterone by $3 a-\mathrm{HSD} 3$. In mice, galeterone is also metabolized to D4G, as well as to three $5 a$-reduced and three $5 \beta$-reduced metabolites (FIG. 1). In 2016, the same group reported that abiraterone is also metabolized into $\Delta 4$-abiraterone (D4A), $5 a$-abiraterone, and five other steroidal metabolites $^{2}$. Abiraterone is a specific inhibitor of CYP17A1, but the D4A metabolite blocks not only CYP17A1 but also 3 $\beta$-HSD and SRD5A, and also acts as a potent AR antagonist. The further $5 \alpha$-reduced or $5 \beta$-reduced metabolites of D4A have been detected in patient sera and 3 -keto- $5 \alpha$-abiraterone ( $5 \alpha \mathrm{A})$, which is present at high concentrations in patient sera, was shown to have AR-agonistic properties.

The first important finding of Alyamani et al. ${ }^{1}$ is that $\mathrm{D} 4 \mathrm{~A}$ is $\sim 100$-fold more potent in inhibiting CYP17A1 than galeterone or any of its metabolites in a cell culture assay. In contrast to abiraterone, galeterone is thought to be an AR degrader, and Alyamani et al. ${ }^{1}$ also observed this property for D4G and $5 \mathrm{aG}$ (the latter only in LNCaP cells); however, as this degradation was only seen at $10 \mu \mathrm{M}$ concentrations of both $\mathrm{D} 4 \mathrm{G}$ and $5 \mathrm{aG}$, the clinical relevance of this observation remains unclear. In addition to its functions as a CYP17A1 inhibitor and AR degrader, galeterone is also an AR antagonist $^{7}$. Importantly, the 3-keto structure of the galeterone metabolites is shared with testosterone and dihydrotestosterone, whereas the $5 a$-reduced metabolites are planar, similar to the more potent dihydrotestosterone. 


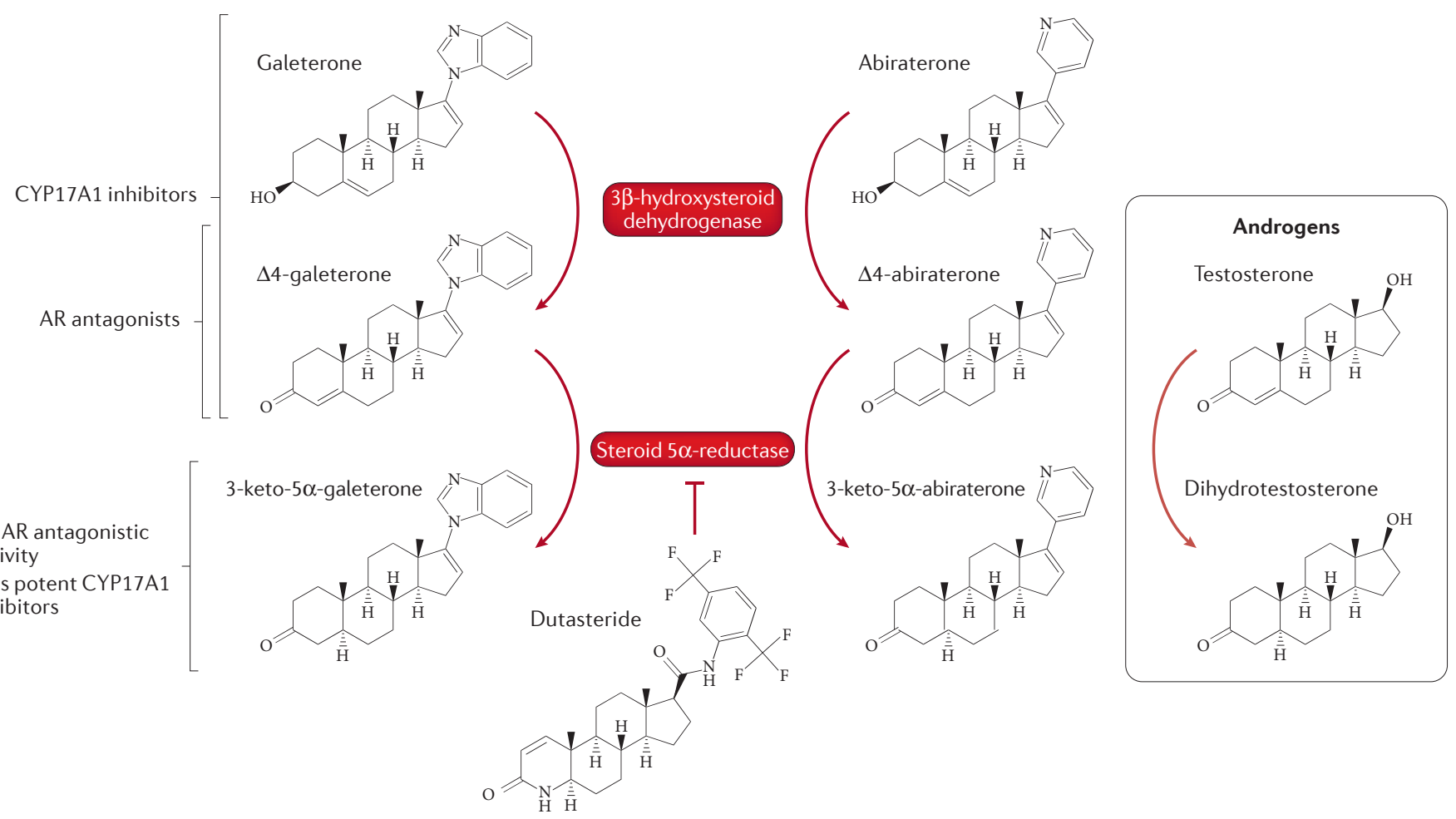

Figure 1 | CYP17A1 inhibitor metabolites and their properties. Galeterone and abiraterone are metabolized into compounds with differing activities on CYP17A1 and the androgen receptor (AR) signalling pathway, owing to their steroidal structure. Dutasteride inhibits steroid $5 a$-reductase and conversion of $\Delta 4$-abiraterone, increasing $\Delta 4$-abiraterone serum levels and decreasing levels of metabolites with possible tumour-promoting properties.
The D4G and $5 a \mathrm{G}$ metabolites have an apparent affinity for the wild-type AR that is similar to that of D4A, whereas galeterone itself has a somewhat lower affinity. Comparisons of the effects of the different metabolites on the expression of androgen-responsive genes in LNCaP and $\mathrm{VCaP}$ cells showed that the properties of galeterone and $\mathrm{D} 4 \mathrm{G}$ are very similar to those of $\mathrm{D} 4 \mathrm{~A}$, whereas $5 \mathrm{aG}$ is a less potent AR inhibitor than its precursors and even showed some partial agonistic activities in LNCaP cells (similar to $5 \alpha \mathrm{A}$, as shown in the 2016 paper $)^{1,2}$.

Interestingly, combined treatment with abiraterone and the SRD5A inhibitor dutasteride in patients with prostate cancer resulted in an increase in serum D4A levels and a decrease in further metabolites with tumour-promoting properties, demonstrating a way to optimize therapy with steroidal CYP17A inhibitors and/or AR antagonists ${ }^{2}$. Whether combining galeterone with dutasteride would also prevent galeterone conversion into a less active CYP17A1 inhibitor or AR-agonistic $5 a \mathrm{G}$ metabolite in patients remains to be investigated.

Patient samples were not available for the study by Alyamani et al. ${ }^{1}$ so metabolism of galeterone was studied only in cell lines and rodents, possibly limiting the clinical relevance of the findings. The enzymes involved in steroid metabolism are highly conserved across species, but their relative activities, expression patterns, and regulation can differ substantially, explaining the differences in serum levels of steroids between men and rodents ${ }^{8}$. In addition, mice lack the high serum concentrations of sex-hormone-binding globulin seen in humans, which has an important effect on the bioavailability and degradation of binding steroids ${ }^{9}$. In conclusion, the metabolic pathways described for abiraterone ${ }^{10}$ and for galeterone ${ }^{1}$ might suggest not only a mechanism behind the lower clinical efficacy of galeterone compared with abiraterone but also a possibly universal problem for the clinical use of steroidal drugs with a similar $\Delta 5,3 \beta$-hydroxyl structure.

Frank Claessens is at the Molecular Endocrinology Laboratory, Department of Cellular and Molecular Medicine, Campus Gasthuisberg, Herestraat 49, PO Box 901, 3000 Leuven, Belgium.

Lisa Moris is at the Molecular Endocrinology Laboratory, Department of Cellular and Molecular Medicine, Campus Gasthuisberg, Herestraat 49, PO Box 901, 3000 Leuven, Belgium; and at the Department of Urology, University Hospitals Leuven, Herestraat 49, 3000 Leuven, Belgium.
Correspondence to F.C. frank.claessens@kuleuven.be doi:10.1038/nrurol.2017.132 Published online 8 Aug 2017

1. Alyamani, M. et al. Steroidogenic metabolism of galeterone reveals a diversity of biochemical activities. Cell Chem. Biol. 24, 825-832.e6 (2017).

2. Li, Z. et al. Redirecting abiraterone metabolism to finetune prostate cancer anti-androgen therapy. Nature 533, 547-551 (2016).

3. Attard, G., Belldegrun, A. S. \& de Bono, J. S. Selective blockade of androgenic steroid synthesis by novel lyase inhibitors as a therapeutic strategy for treating metastatic prostate cancer. BJU Int. 96, 1241-1246 (2005).

4. Yin, L. \& Hu, Q. CYP17 inhibitors - abiraterone, C17,20-lyase inhibitors and multi-targeting agents. Nat. Rev. Urol. 11, 32-42 (2014).

5. James, N. D. et al. Abiraterone for prostate cancer not previously treated with hormone therapy. N. Engl. J. Med. http://dx.doi.org/10.1056/NEJMoa1702900 (2017).

6. Handratta, V. D. et al. Novel C-17-heteroaryl steroidal CYP17 inhibitors/antiandrogens: synthesis, in vitro biological activity, pharmacokinetics, and antitumor activity in the LAPC4 human prostate cancer xenograft model. J. Med. Chem. 48, 2972-2984 (2005).

7. Yu, Z. et al. Galeterone prevents androgen receptor binding to chromatin and enhances degradation of mutant androgen receptor. Clin. Cancer Res. 20, 4075-4085 (2014).

8. Nilsson, M. E. et al. Measurement of a comprehensive sex steroid profile in rodent serum by high-sensitive gas chromatography-tandem mass spectrometry. Endocrinology 156, 2492-2502 (2015).

9. Laurent, M. R. et al. Sex hormone-binding globulin regulation of androgen bioactivity in vivo: validation of the free hormone hypothesis. Sci. Rep. 6, 35539 (2016)

10. Li, Z. et al. Conversion of abiraterone to D4A drives anti-tumour activity in prostate cancer. Nature $\mathbf{5 2 3}$ 347-351 (2015).

Competing interests statement

The authors declare no competing interests. 